\title{
GPR143 is a Prognostic Biomarker Associated with Immune Infiltration in Melanoma
}

\author{
Zixuan Xing ( $\sim 15771193655 @ 163 . c o m$ ) \\ Xi'an Jiaotong University School of Medicine \\ Shaobo Wu \\ Xi'an Jiaotong University \\ Qijuan Zang \\ Xi'an Jiaotong University School of Medicine \\ Hao Lei \\ Xi'an Jiaotong University Second Affiliated Hospital \\ Yi Wei \\ Xi'an Jiaotong University Medical College First Affiliated Hospital \\ Chaonan Zhang \\ Xi'an Jiaotong University Medical College First Affiliated Hospital \\ Bingling Dai \\ Xi'an Jiaotong University School of Medicine
}

\section{Research Article}

Keywords: GPR143, SKCM, Immune infiltration, Biomarker, TCGA, Bioinformatics

Posted Date: May 4th, 2021

DOI: https://doi.org/10.21203/rs.3.rs-430494/v1

License: @ (i) This work is licensed under a Creative Commons Attribution 4.0 International License. Read Full License 


\section{Abstract}

Background: Skin cutaneous melanoma (SKCM) is considered one of the most aggressive and lethal cancers of the skin. G-protein coupled receptor 143 (GPR143), which has been reported to cause congenital nystagmus, belongs to the superfamily of G proteincoupled receptors.

Methods and Results: We analyzed the expression of GPR143 and survival of SKCM patients in SKCM via Gene Expression Profiling Interactive Analysis (GEPIA). Then, logistic regression and multivariate cox analysis was used to analyze the influence of GPR143 expression on clinicopathological elements and survival. We explored the immune response of 22 TIICs in SKCM via CIBERSORT and used TIMER to assess the correlation of GPR143 expression and immune infiltration level. Finally, we used gene set enrichment analysis (GSEA) to assess the TCGA dataset. The outcomes suggest that GPR143 expression in tumor samples is remarkedly higher than in normal samples and high GPR143 expression is associated with poorer prognosis. The result of multivariate analysis indicated that increased GPR143 expression is an independent prognostic factor for prognosis. We found GPR143 expression level has prominent negative correlations with infiltrating levels of B cell, CD8+ T cells, etc. GSEA indicated that pigment metabolic process, pigment biosynthetic process and other pathways were identified as differentially enriched pathways in Gene Ontology (GO). Oxidative phosphorylation, Parkinson's disease and other pathways were showed significantly differential enrichment in GPR143 high expression phenotype in Kyoto Encyclopedia of Genes and Genomes (KEGG).

Conclusions: In conclusion, GPR143 may be a novel prognostic biomarker and associated with immune infiltrates in SKCM.

\section{Introduction}

Skin cutaneous melanoma (SKCM) is considered one of the most aggressive and lethal cancers of the skin [1-3]. Among skin tumors, melanoma is frequently characterized by its poor prognosis and low survival rate [4-6]. To make things worse, there are no effective systemic therapies to treat advanced stages of SKCM $[4,7,8]$. The main prognostic indicators following diagnosis of cutaneous melanoma can be divided into histological and clinical factors $[9,10]$. Recently, five chemokine members (CXCL9, CXCL10, CXCL13, CCL4, CCL5) was proved to be associated with SKCM tumorigenesis, progression, prognosis and immune infiltrations[11]. However, we need to find better biomarkers.

G-protein coupled receptor 143 (GPR143) belongs to the superfamily of G protein-coupled receptors. Ocular albinism (OA) can be caused by mutations in GPR143, which is located on the X-chromosome [12]. The GPR143 gene, which has also been reported to cause congenital nystagmus, possesses nine exons and spans $\sim 40 \mathrm{~kb}$ of genomic DNA [13]. GPR143 encodes proteins that are important for the development and maturation of melanosomes, and is only localized in the lysosomes and melanosomes of cells [13]. In Skin cutaneous melanoma, only a little information has been made available about GPR143.

In order to explore the role of GPR143 in SKCM, we conducted a further analysis using the data downloaded from The Cancer Genome Atlas (TCGA). Moreover, the correlation of GPR143 with SKCM was identified by Gene Expression Profiling Interactive Analysis (GEPIA), the $R$ and COX regression analysis. Meanwhile, CIBERSORT, a recent metagene approach, was used along with the Tumor Immune Estimation Resource (TIMER) to test the relative proportions of different types of tumor infiltrating immune cells (TIICS) in various tumor microenvironments. In addition, these methods helped us to study the association of GPR143 with tumorinfiltrating immune cells. The results allowed us to have a comprehensive understanding for the potential function of GPR143 in SKCM. Also, the findings demonstrated a possible correlation as well as a latent mechanism between GPR143 and tumor-immune interactions. Therefore, GPR143 may become a novel sign for the evaluation of prognosis and immune infiltration for SKCM patients.

\section{Materials And Methods}

\section{Data acquisition}

SKCM patient datasets, with gene expression profiles and clinical information, were downloaded from the publicly available TCGA (https://TCGAData.nci.nih.gov/TCGA/) [14], and included 1 normal and 471 tumor tissues. Subsequent processing excluded cases with insufficient or missing data on age, local invasion, lymph node metastasis, distant metastasis, TNM stage and overall survival time. Then, we transformed RNA sequencing data to convert count data to value more similar to those from microarrays. 
Furthermore, in light of the expression of GPR143 in further study, tumor tissues were divided into 2 groups to study the effects of GPR143 expression on immune microenvironment.

\section{Survival and expression analysis by GEPIA}

The correlation between GPR143 expression and clinicopathologic information in SKCM was confirmed by Gene Expression Profiling Interactive Analysis (GEPIA) (http:// gepia.cancer-pku.cn/index.html). In GEPIA[15], a standard processing pipeline was used to analyzes 9,736 tumors specimens from the RNA sequencing expression data and 8,587 normal samples from GTEx and TCGA. The correlation of the gene expression with SKCM patients' prognosis could be found by the analysis of survival curve of differential GPR143 expression in GEPIA. Moreover, stage plot that used pathological stage as variable was analyzed to compare GPR143 expression in various pathological stage. Additionally, boxplots that used disease state (Tumor or Normal) as variable was drew to show differential expression of GPR143.

\section{Evaluation of tumor-infiltrating immune cells}

TIMER [16] as a comprehensive resource (https://cistrome.shinyapps.io/timer/) was employed in the systematic analysis of immune infiltrates across a wide spectrum of cancer types. A novel statistical approach applied by TIMER called the deconvolution using the gene expression profiles [17] to deduce an effect on the number of tumor-infiltrating immune cells (TIICs). The Cancer Genome Atlas provided the TIMER database 10,897 specimens across 32 cancer types applied for the approximate calculation of immune infiltrates. A sequence of analysis was conducted on the expression of GPR143 in various types of cancer and its correlation with plentiful immune infiltrates. B cells, CD4+ T cells, CD8+ T cells, macrophages, neutrophils, and dendritic cells was included in these immune infiltrates.

CIBERSORT [18] (http://cibersort.stanford.edu/), an online analytical platform based on a deconvolution algorithm, was used to assess the relevance of correlation between TIICs in tumor and gene expression. Thus, CIBERSORT could be used to precisely estimate TIIC concentration. In order to analyze its correlation with survival and molecular subpopulation, we evaluated the immune response of 22 TIICs in SKCM via CIBERSORT. Meanwhile, 472 samples from the TCGA were used to assess the influence of GPR143 expression. Also, 472 samples were divided into two groups, low expression group and high expression group, and then we used the data to make vioplot.

\section{Gene set enrichment analysis}

A computational method that determined the statistical significance of a priori defined set of genes and the existence of concordant differences between two biological states was known as the GSEA [19, 20]. Gene set enrichment analysis (GSEA), a computational method, assessed whether a specific gene set is conspicuous different in any two biological states. In our study, in light of their correlation with the GPR143 expression, a list was firstly generated on the classification of the genes. This computational method expounded on the prominent differences on the survival between high- and low- GPR143 groups. In addition, in order to sort the enriched pathways in each phenotype, we used the nominal $P$ value and normalized enrichment score (NES) [21]. Those gene set with a discovery rate $(F D R)<0.05$ were deemed to be markedly enriched.

\section{Statistical analysis}

Data acquired from TCGA were merged and processed by R3.6.3. The correlations between the clinical information and GPR143 expression were assessed by using logistic regression. Furthermore, Multivariate Cox analysis was used to analyze the influence of GPR143 expression and other clinicopathological elements (age and gender) on survival. $P$ value $<0.05$ was set up as the cut-off criterion. To study the correlation between 22 types of immune cells, we conducted the correlation heatmap to show the correlation between every two different immune cells in specimens.

\section{Results}




\section{Patient characteristics and multivariate analysis}

Our study cohort represented SKCM with tumor stages of I and II, where stage I embodied 77 patients (35.5\%) and stage II encompassed 140 (64.5\%).

Survival curve, pathological stage plot and expression on boxplot conducted by GEPIA were shown in Fig. 1 and Fig. 2. It suggested that increased expression of GPR143 was significantly associated with different disease states (Tumor or Normal) $(P<0.05)$ and overall survival $(P<0.05)$. Additionally, GPR143 expression in tumor samples was remarkedly higher than in normal samples. In Univariate analysis (Table 1$)$, Some factors, including age $(\mathrm{HR}=1.02, P$ value $<0.001)$, pathological stage $(\mathrm{HR}=1.57, P$ value $<0.001)$ along with the expression of GPR143 $(\mathrm{HR}=1.00, P$ value $=0.001)$ were significantly correlated with overall survival. As shown in Fig. 3, multivariate analysis of correlation of using Cox regression revealed that $\mathrm{T}$ (Stage of primary tumor), $\mathrm{N}$ (Stage of regional lymph node) and GPR143 expression were independent prognostic factors.

Table 1

(A) Association with overall survival and clinicopathologic characteristic in TCGA patients using Cox regression. (B) Multivariate survival using Cox regression.

\begin{tabular}{|lll|}
\hline Clinical characteristics & $\mathrm{HR}(\mathbf{9 5} \% \mathrm{Cl})$ & p-Value \\
\hline A & & \\
\hline Age & $1.02(1.01-1.03)$ & 0.000 \\
\hline Gender & $1.05(0.74-1.48)$ & 0.794 \\
\hline Stage & $1.57(1.29-1.91)$ & 0.000 \\
\hline GPR143 & $1.00(1.00-1.01)$ & 0.001 \\
\hline B & & \\
\hline Stage & $0.82(0.57-1.19)$ & 0.298 \\
\hline GPR143 & $1.00(1.00-1.01)$ & 0.041 \\
\hline
\end{tabular}

According to Table 1, Fig. 2 and Fig. 3, GPR143 expression associated significantly with tumor stage. Meanwhile, with the growth of tumor stage, GPR143 expression increased obviously.

\section{Relationship between GPR143 expression and tumour-infiltrating immune cells}

In the past, analyses suggested tumor-infiltrating lymphocytes as independent predictors of sentinel lymph node status and survival in cancer patients [22]. Hence, we analyzed whether GPR143 expression has a correlation with immune infiltration in SKCM. 471 tumor samples were divided into 2 parts according to GPR143 expression. An established computational resource (CIBERSORT) was utilized to explore gene expression profiles of downloaded samples to analyze the density of 22 types of immune cells. The resulted of CIBERSORT (Fig. 4A) exhibited the proportion of 22 subpopulations of immune cells helped assess their concentrations in the high and low GPR143 expression groups. T cells CD4 memory resting, T cells regulatory (T-regs) and NK cells activated were main immune cells effected by GPR143 expression. Among them, T cells CD4 memory resting $(P=0.003)$, was apparently decreased in high expression group compared with low expression group. In contrast, T cells regulatory (T-regs) $(P=0.008)$, NK cells activated $(P=$ 0.005) were increased in high expression group compared with low expression group. What's more, as shown in Fig. 4B, correlation heatmap revealed that the proportions of different TIICs subpopulations were weakly to moderately correlated.

\section{GPR143 expression was correlated with immune infiltration level in SKCM and cumulative survival in SKCM from TIMER}


Previous analyses have demonstrated that tumor-infiltrating lymphocyte grade was an independent predictor of sentinel lymph node status and survival [22]. Therefore, we investigated how, in different types of cancer, the GPR143 expression was associated with immune infiltration levels. Also, we assessed the correlations of GPR143 expression with immune infiltration levels via TIMER. In our selection of different types of cancer, we found that GPR143 were positively associated with tumor purity by selecting expression levels of GPR143. The results showed the correlation of GPR143 expression level with poorer prognosis and high immune infiltration in SKCM. Furthermore, a negative correlation existed between the GPR143 expression level and infiltrating levels of $B$ cell $(r=-0.124$, $P=8.70 \mathrm{e}-03), \mathrm{CD} 8+\mathrm{T}$ cells $(\mathrm{r}=-0.219, P=3.59 \mathrm{e}-06), \mathrm{CD} 4+\mathrm{T}$ cells $(\mathrm{r}=-0.043, P=3.63 \mathrm{e}-01)$, Macrophages $(\mathrm{r}=-0.214, P=4.46 \mathrm{e}-06)$, Neutrophils ( $r=-0.31, P=1.67 \mathrm{e}-11)$, and DCs $(r=-0.185, P=8.88 \mathrm{e}-05)$ in SKCM (Fig. 5A). The B cell, CD8 + T cells, Neutrophils, and DCs were factors related to the cumulative survival rate of SKCM over time (Fig. 5B).

\section{Gene sets enriched in GPR143 expression phenotype}

GPR143-related signaling pathways based on GSEA was used to identify signaling pathways involved in SKCM between low and high GPR143 expression data sets, and demonstrated significant differences (FDR $<0.05$, NOM $P$ value $<0.05)$ in enrichment of GO and KEGG. Owing to the limited space, only nine pathways of high and eight pathways of low expression were listed here (Table 2 and Table 3). 4 KEGG items including oxidative phosphorylation, Parkinson's disease, Alzheimer's disease and Citrate cycle (TCA cycle) were showed significantly differential enrichment in GPR143 high expression phenotype and 4 KEGG items including hypertrophic cardiomyopathy (HCM), JAK STAT signaling pathway, systemic lupus erythematosus and Cytokine-cytokine receptor interaction were showed significantly differential enrichment in GPR143 low expression phenotype based on NES, NOM $P$ value, and FDR value (Fig. 6A). $5 \mathrm{GO}$ items including pigment metabolic process, pigment biosynthetic process, developmental pigmentation, melanin metabolic process and pigment cell differentiation were showed significantly differential enrichment in GPR143 high expression phenotype. $4 \mathrm{GO}$ items including negative regulation of CD4 positive alpha beta $\mathrm{T}$ cell activation, negative regulation of $\mathrm{T}$ helper cell differentiation, negative regulation of CD4 positive alpha beta $T$ cell differentiation and negative regulation of alpha beta $T$ cell activation were showed significantly differential enrichment in GPR143 low expression phenotype based on NES, NOM $P$ value, and FDR value (Fig. 6B). Those all indicating the potential role of GPR143 in the development of SKCM.

Table 2

Gene sets enriched in GPR143 high expression phenotype.

\begin{tabular}{|llll|}
\hline Gene set name & NES & NOM p-val & FDR q-val \\
\hline KEGG_OXIDATIVE_PHOSPHORYLATION & 2.339681 & 0 & 0 \\
\hline KEGG_PARKINSONS_DISEASE & 2.168304 & 0 & 0.001657 \\
\hline KEGG_ALZHEIMERS_DISEASE & 2.107668 & 0.002062 & 0.003162 \\
\hline KEGG_CITRATE_CYCLE_TCA_CYCLE & 2.069325 & 0.002058 & 0.004536 \\
\hline GO_PIGMENT_METABOLIC_PROCESS & 2.558049 & 0 & 0 \\
\hline GO_PIGMENT_BIOSYNTHETIC_PROCESS & 2.475338 & 0 & 0 \\
\hline GO_DEVELOPMENTAL_PIGMENTATION & 2.330577 & 0 & $3.76 \mathrm{E}-05$ \\
\hline GO_MELANIN_METABOLIC_PROCESS & 2.258216 & 0 & $1.87 \mathrm{E}-04$ \\
\hline GO_PIGMENT_CELL_DIFFERENTIATION & 2.162642 & 0 & $9.00 \mathrm{E}-04$ \\
\hline
\end{tabular}


Table 3

Gene sets enriched in GPR143 low expression phenotype.

\begin{tabular}{|c|c|c|c|}
\hline Gene set name & NES & $\begin{array}{l}\text { NOM p- } \\
\text { val }\end{array}$ & $\begin{array}{l}\text { FDR q- } \\
\text { val }\end{array}$ \\
\hline KEGG_HYPERTROPHIC_CARDIOMYOPATHY_HCM & -1.97406 & 0.002101 & 0.025692 \\
\hline KEGG_JAK_STAT_SIGNALING_PATHWAY & -1.92468 & 0.001984 & 0.026611 \\
\hline KEGG_SYSTEMIC_LUPUS_ERYTHEMATOSUS & -1.92975 & 0.006012 & 0.028755 \\
\hline KEGG_CYTOKINE_CYTOKINE_RECEPTOR_INTERACTION & -1.98046 & 0.007843 & 0.0316 \\
\hline GO_NEGATIVE_REGULATION_OF_CD4_POSITIVE_ALPHA_BETA_T_CELL_ACTIVATION & -2.13033 & 0 & 0.016592 \\
\hline GO_NEGATIVE_REGULATION_OF_T_HELPER_CELL_DIFFERENTIATION & -2.12384 & 0 & 0.01716 \\
\hline GO_NEGATIVE_REGULATION_OF_CD4_POSITIVE_ALPHA_BETA_T_CELL_DIFFERENTIATION & -2.09987 & 0 & 0.017953 \\
\hline GO_NEGATIVE_REGULATION_OF_ALPHA_BETA_T_CELL_ACTIVATION & -2.13735 & 0 & 0.018617 \\
\hline
\end{tabular}

\section{Data validation}

The Human Protein Atlas (HPA, http://www.proteinatlas.org) was a database that contains transcriptomes, proteomes and immunohistochemistry-based expression data [23]. The HPA database provided abundant data of transcriptome and proteomes in specific human tissues through RNA-sequencing analysis and immunohistochemistry analysis [24]. Immunohistochemistry analysis available from the HPA showed that in tumor tissues, GPR143 has higher levels of expression when compared to normal tissues (Fig. 7).

\section{Discussion}

GPR143 is a gene reported in X-linked ocular albinism type 1 [13]. We found that variations in GPR143 expression level relate to prognosis in SKCM. According to our knowledge, only a few literatures had reported the potential prognostic impact of GPR143 in SKCM. As a result, in our study, we focused on the potential role of GPR143 in SKCM that conducted the first-ever analysis on the GPR143 expression in a large cohort of SKCM patients. Based on the clinical and RNA-seq data, we conducted a retrospective analysis of 471 cases of SKCM patients and confirmed it with histology. The outcome of our study highlighted that in melanoma patients, the GPR143 expression is correlated with the tumor grade.

In this study, the result demonstrated that variations in GPR143 expression levels were associated with the prognosis in SKCM. High GPR143 expression has a correlation with a positive prognosis in SKCM. Meanwhile, GPR143 expression is an independent prognostic factor for prognosis. We found the correlation of GPR143 expression with survival of SKCM patients via GEPIA. The upregulated expression of GPR143 is correlated with the negative prognosis. We also observed that normal tissues and tumor tissues of SKCM had different GPR143 expression. The underlying mechanisms and relationships of GPR143 expression in cancer needed to be studied further. Hence, we downloaded datasets from TCGA and then utilized R3.6.3 to assess the relationships between GPR143 and tumor stages. Multivariate analysis of correlation of using Cox regression revealed that GPR143 expression was an independent prognostic factor of SKCM patient's prognosis.

Furthermore, the results revealed that the extent of immune infiltration levels and the diversity of immune marker sets were correlated with GPR143 expression in SKCM. Thus, we suggested that GPR143 may have a potential effect on tumor immunology and can be used as a promising biomarker for cancer diagnosis. In our study, we noticed a significant conclusion that the GPR143 expression has a significant correlation with immune infiltration levels in SKCM. A CIBERSORT analysis revealed a substantial positive association of GPR143 expression with infiltration levels of T cells regulatory (T-regs), NK cells activated in SKCM. Likewise, the conclusion that GPR143 regulates tumor immune microenvironment of SKCM is reflected in the relationships between gene marker of diverse immune cells and GPR143 expression. The tumor environment is composed of tumor cells, stromal cells and tumor- 
infiltrating immune cells of both innate and adaptive lineages [25-27]. The composition of tumor infiltrating immune cells can vary according to cancer type [28-30]. T cell-infiltrating tumors display phenotypic and functional features of exhausted T cells, indicating that they are functionally impaired due to tumor antigen overload and various tumor immune escape mechanisms [31-33]. Immune Cell Infiltration because ICls activate the immune response to cancer, infiltration of immune cells, including $T$ cells, into tumors may induce tumor regression following treatment [34,35]. Thus, the potential influence of GPR143 on SKCM may relates to the high density of immune cells. In addition, we found a negative correlation exists between the GPR143 expression level and infiltrating levels of B cell $(r=-0.124, P=8.70 \mathrm{e}-03)$, CD8 + T cells $(r=-0.219, P=3.59 \mathrm{e}-06)$, CD $4+\mathrm{T}$ cells $(\mathrm{r}=-0.043, P=3.63 \mathrm{e}-01)$, Macrophages $(r=-0.214, P=4.46 \mathrm{e}-06)$, Neutrophils $(r=-0.31, P=1.67 \mathrm{e}-11)$, and DCs $(r=-0.185, P=8.88 \mathrm{e}-05)$ in SKCM.

To further study the functions of GPR143 in SKCM, we used TCGA data with GSEA. GSEA indicated that oxidative phosphorylation, Parkinson's disease, Alzheimer's disease and Citrate cycle (TCA cycle) in KEGG and pigment metabolic process, pigment biosynthetic process, developmental pigmentation, melanin metabolic process and pigment cell differentiation in $\mathrm{GO}$ are differentially enriched in GPR143 high expression phenotype. Those all suggested that GPR143 may be a therapeutic target and potential prognostic indicator of SKCM.

GPR143 is a Protein Coding gene and is exclusively expressed by pigment cells [36]. The mechanism of GPR143 in these cells remains unclear. However, some studies have proved that GPR143 is significantly associated to the occurrence and development of SKCM. One study had proved that GPR143 is the highest up-regulated GPCR in SKCM [37]. Even though there is no clear explanation for the correlation between GPR143 and SKCM, based on our research results and previous studies, we have reason to believe that GPR143 play a crucial role in the progression of SKCM and will affect the development of pathophysiological mechanism of SKCM.

In summary, GPR143 likely has an important influence on immune infiltration and serve as a probable prognostic molecular marker of SKCM. Moreover, pigment metabolic process, pigment biosynthetic process, developmental pigmentation, melanin metabolic process and pigment cell differentiation are the possible key pathways regulated by GPR143 in SKCM. Finally, the findings further reveal that we could use the high GPR143 expression as an independent prognostic factor for prognosis in SKCM patients.

\section{Conclusion}

G-protein coupled receptor 143 (GPR143) belongs to the superfamily of G protein-coupled receptors. It has been reported that GPR143 is related to the occurrence and development of some diseases, such as congenital nystagmus[13]. In this study, we used GEPIA data to analyze the correlation between the expression of GPR143 and SKCM in clinical and immunology and proved that GPR143 can be used as an independent prognostic factor and it's associated with immune infiltration. Our research has potential significance for finding new prognostic factors for SKCM.

\section{Declarations}

\section{Acknowledgements:}

We would like to thank all the participants and research staffs in School of Pharmacy, Health Science Center, Xi'an Jiaotong University for their invaluable contributions to this work.

\section{Funding:}

Our study was supported by the Fundamental Research Funds for the Central Universities (xjj2018167), the National Science Foundation for Post-doctoral Scientists of China (Grant no. 2019M653670) and Natural Science Basic Research Plan in Shan'xi Province of China (Grant no. 2019JQ-596).

\section{Conflicts of Interest:}

Declarations of interest: none. 


\section{Availability of data and material:}

Not applicable.

\section{Code availability:}

Not applicable.

\section{Author contribution:}

BD, ZX and SW conceived and designed the study. ZX, SW, QZ, LH analyzed the data. WY and CZ carried out the statistics. ZX and SW drafted the manuscript. QZ, LH, WY and CZ revised the manuscript. All authors have read, commented on, and approved the manuscript.

\section{Ethics approval:}

Not applicable.

\section{Consent to Participate (Ethics):}

Not applicable.

\section{Consent to Publish (Ethics):}

Authors approve for submitting the publication.

\section{References}

1. Berge LAM, Andreassen BK, Stenehjem JS, Heir T, Furu K, Juzeniene A, et al. Use of Antidepressants and Risk of Cutaneous Melanoma: A Prospective Registry-Based Case-Control Study. Clin Epidemiol. 2020;12:193-202. doi: 10.2147/clep.S241249.

2. Wang Q, Pan F, Li S, Huang R, Wang X, Wang S, et al. The prognostic value of the proteasome activator subunit gene family in skin cutaneous melanoma. J Cancer. 2019;10(10):2205-19. doi: 10.7150/jca.30612.

3. Zhang L, Wang Q, Wang L, Xie L, An Y, Zhang G, et al. OSskcm: an online survival analysis webserver for skin cutaneous melanoma based on 1085 transcriptomic profiles. Cancer Cell Int. 2020;20:176. doi: 10.1186/s12935-020-01262-3.

4. Alvarez-Artime A, Cernuda-Cernuda R, Francisco Artime N, Cepas V, Gonzalez-Menendez P, Fernadez-Vega S, et al. MelatoninInduced Cytoskeleton Reorganization Leads to Inhibition of Melanoma Cancer Cell Proliferation. Int J Mol Sci. 2020;21(2). doi: 10.3390/ijms21020548.

5. Li J, Zhang Z, Li G. Patient outcome prediction using multiple biomarkers in human melanoma: A clinicopathological study of 118 cases. Exp Ther Med. 2011;2(1):131-5. doi: 10.3892/etm.2010.169.

6. Wang Y, Li D, Lu J, Chen L, Zhang S, Qi W, et al. Long noncoding RNA TTN-AS1 facilitates tumorigenesis and metastasis by maintaining TTN expression in skin cutaneous melanoma. Cell Death Dis. 2020;11(8):664. doi: 10.1038/s41419-020-02895-y.

7. Lai C, Duan S, Ye F, Hou X, Li X, Zhao J, et al. The enhanced antitumor-specific immune response with mannose- and CpG-ODNcoated liposomes delivering TRP2 peptide. \#N/A. 2018;8(6):1723-39. doi: 10.7150/thno.22056.

8. Murekatete B, Shokoohmand A, McGovern J, Mohanty L, Meinert C, Hollier BG, et al. Targeting Insulin-Like Growth Factor-I and Extracellular Matrix Interactions in Melanoma Progression. Sci Rep. 2018;8(1):583. doi: 10.1038/s41598-017-19073-4.

9. Vilanova CM, Lages RB, Ribeiro SM, Almeida IP, Santos LG, Vieira SC. Epidemiological and histopathological profile of cutaneous melanoma at a center in northeastern Brazil from 2000 to 2010. An Bras Dermatol. 2013;88(4):545-53. doi: 10.1590/abd18064841.20132036. 
10. Dinnes J, Ferrante di Ruffano L, Takwoingi Y, Cheung ST, Nathan P, Matin RN, et al. Ultrasound, CT, MRI, or PET-CT for staging and re-staging of adults with cutaneous melanoma. \#N/A. 2019;7(7):Cd012806. doi: 10.1002/14651858.CD012806.pub2.

11. Huang B, Han W, Sheng ZF, Shen GL. Identification of immune-related biomarkers associated with tumorigenesis and prognosis in cutaneous melanoma patients. Cancer Cell Int. 2020;20:195. doi: 10.1186/s12935-020-01271-2.

12. Grønskov K, Jespersgaard C, Bruun GH, Harris P, Brøndum-Nielsen K, Andresen BS, et al. A pathogenic haplotype, common in Europeans, causes autosomal recessive albinism and uncovers missing heritability in OCA1. Sci Rep. 2019;9(1):645. doi: 10.1038/s41598-018-37272-5.

13. Gao X, Liu T, Cheng X, Dai A, Liu W, Li R, et al. A novel GPR143 mutation in a Chinese family with X-linked ocular albinism type 1. Mol Med Rep. 2020;21(1):240-8. doi: 10.3892/mmr.2019.10813.

14. Blum A, Wang P, Zenklusen JC. SnapShot: TCGA-Analyzed Tumors. Cell. 2018;173(2):530. doi: 10.1016/j.cell.2018.03.059.

15. Tang Z, Li C, Kang B, Gao G, Li C, Zhang Z. GEPIA: a web server for cancer and normal gene expression profiling and interactive analyses. Nucleic Acids Res. 2017;45(W1):W98-w102. doi: 10.1093/nar/gkx247.

16. Li T, Fan J, Wang B, Traugh N, Chen Q, Liu JS, et al. TIMER: A Web Server for Comprehensive Analysis of Tumor-Infiltrating Immune Cells. Cancer Res. 2017;77(21):e108-e10. doi: 10.1158/0008-5472.Can-17-0307.

17. Li B, Severson E, Pignon JC, Zhao H, Li T, Novak J, et al. Comprehensive analyses of tumor immunity: implications for cancer immunotherapy. Genome Biol. 2016;17(1):174. doi: 10.1186/s13059-016-1028-7.

18. Gentles AJ, Newman AM, Liu CL, Bratman SV, Feng W, Kim D, et al. The prognostic landscape of genes and infiltrating immune cells across human cancers. Nat Med. 2015;21(8):938-45. doi: 10.1038/nm.3909.

19. Subramanian A, Kuehn H, Gould J, Tamayo P, Mesirov JP. GSEA-P: a desktop application for Gene Set Enrichment Analysis. Bioinformatics. 2007;23(23):3251-3. doi: 10.1093/bioinformatics/btm369.

20. Tu Z, Xiong J, Xiao R, Shao L, Yang X, Zhou L, et al. Loss of miR-146b-5p promotes T cell acute lymphoblastic leukemia migration and invasion via the IL-17A pathway. J Cell Biochem. 2019;120(4):5936-48. doi: 10.1002/jcb.27882.

21. Subramanian A, Tamayo P, Mootha VK, Mukherjee S, Ebert BL, Gillette MA, et al. Gene set enrichment analysis: a knowledgebased approach for interpreting genome-wide expression profiles. Proc Natl Acad Sci U S A. 2005;102(43):15545-50. doi: 10.1073/pnas.0506580102.

22. Azimi F, Scolyer RA, Rumcheva P, Moncrieff M, Murali R, McCarthy SW, et al. Tumor-infiltrating lymphocyte grade is an independent predictor of sentinel lymph node status and survival in patients with cutaneous melanoma. J Clin Oncol. 2012;30(21):2678-83. doi: 10.1200/jco.2011.37.8539.

23. Yang M, Chen H, Zhou L, Chen K, Su F. Expression profile and prognostic values of STAT family members in non-small cell lung cancer. Am J Transl Res. 2019;11(8):4866-80.

24. Gao L, Zhong J-C, Huang W-T, Dang Y-W, Kang M, Chen G. Integrative analysis of BSG expression in NPC through immunohistochemistry and public high-throughput gene expression data. Am J Transl Res. 2017;9(10):4574-92.

25. Liu Y, O'Leary CE, Wang LS, Bhatti TR, Dai N, Kapoor V, et al. CD11b+Ly6G+ cells inhibit tumor growth by suppressing IL-17 production at early stages of tumorigenesis. \#N/A. 2016;5(1):e1061175. doi: 10.1080/2162402x.2015.1061175.

26. Dai X, Luo Y, Xu Y, Zhang J. Key indexes and the emerging tool for tumor microenvironment editing. Am J Cancer Res. 2019;9(5):1027-42.

27. Wang J, Huang F, Jiang C, Chi P. Silencing Signal Transducer and Activator of Transcription 3 (STAT3) and Use of AntiProgrammed Cell Death-Ligand 1 (PD-L1) Antibody Induces Immune Response and Anti-Tumor Activity. Med Sci Monit. 2020;26:e915854. doi: 10.12659/msm.915854.

28. Garcia-Chagollan M, Carranza-Torres IE, Carranza-Rosales P, Guzmán-Delgado NE, Ramírez-Montoya H, Martínez-Silva MG, et al. Expression of NK Cell Surface Receptors in Breast Cancer Tissue as Predictors of Resistance to Antineoplastic Treatment. Technol Cancer Res Treat. 2018;17:1533033818764499. doi: 10.1177/1533033818764499.

29. Xiong Y, Wang Z, Zhou Q, Zeng H, Zhang H, Liu Z, et al. Identification and validation of dichotomous immune subtypes based on intratumoral immune cells infiltration in clear cell renal cell carcinoma patients. J Immunother Cancer. 2020;8(1). doi: 10.1136/jitc-2019-000447.

30. Yang S, Liu T, Cheng Y, Bai Y, Liang G. Immune cell infiltration as a biomarker for the diagnosis and prognosis of digestive system cancer. Cancer Sci. 2019;110(12):3639-49. doi: 10.1111/cas.14216.

Page 9/15 
31. Jeske SS, Schuler PJ, Doescher J, Theodoraki MN, Laban S, Brunner C, et al. Age-related changes in T lymphocytes of patients with head and neck squamous cell carcinoma. Immun Ageing. 2020;17:3. doi: 10.1186/s12979-020-0174-7.

32. Gravelle P, Do C, Franchet C, Mueller S, Oberic L, Ysebaert L, et al. Impaired functional responses in follicular lymphoma CD8(+)TIM-3(+) T lymphocytes following TCR engagement. \#N/A. 2016;5(10):e1224044. doi: 10.1080/2162402x.2016.1224044.

33. Heylmann D, Badura J, Becker H, Fahrer J, Kaina B. Sensitivity of CD3/CD28-stimulated versus non-stimulated lymphocytes to ionizing radiation and genotoxic anticancer drugs: key role of ATM in the differential radiation response. Cell Death Dis. 2018;9(11):1053. doi: 10.1038/s41419-018-1095-7.

34. Nakamura Y. Biomarkers for Immune Checkpoint Inhibitor-Mediated Tumor Response and Adverse Events. Front Med (Lausanne). 2019;6:119. doi: 10.3389/fmed.2019.00119.

35. Hanoteau A, Newton JM, Krupar R, Huang C, Liu HC, Gaspero A, et al. Tumor microenvironment modulation enhances immunologic benefit of chemoradiotherapy. J Immunother Cancer. 2019;7(1):10. doi: 10.1186/s40425-018-0485-9.

36. Naruto T, Okamoto N, Masuda K, Endo T, Hatsukawa Y, Kohmoto T, et al. Deep intronic GPR143 mutation in a Japanese family with ocular albinism. Sci Rep. 2015;5:11334. doi: 10.1038/srep11334.

37. Sriram K, Moyung K, Corriden R, Carter H, Insel PA. GPCRs show widespread differential mRNA expression and frequent mutation and copy number variation in solid tumors. PLoS Biol. 2019;17(11):e3000434. doi: 10.1371/journal.pbio.3000434.

\section{Figures}

A

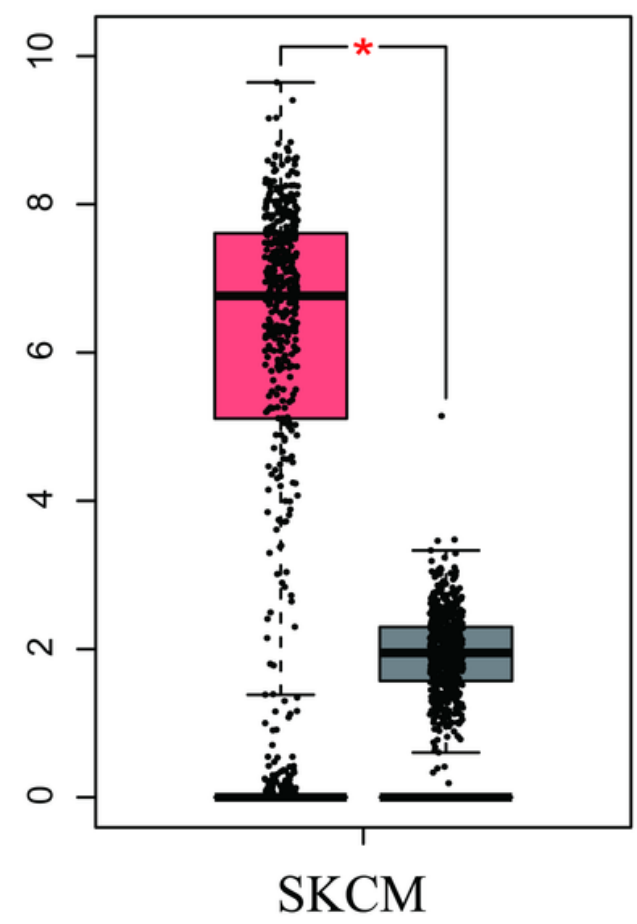

$(\operatorname{num}(\mathrm{T})=461 ; \operatorname{num}(\mathrm{N})=558)$

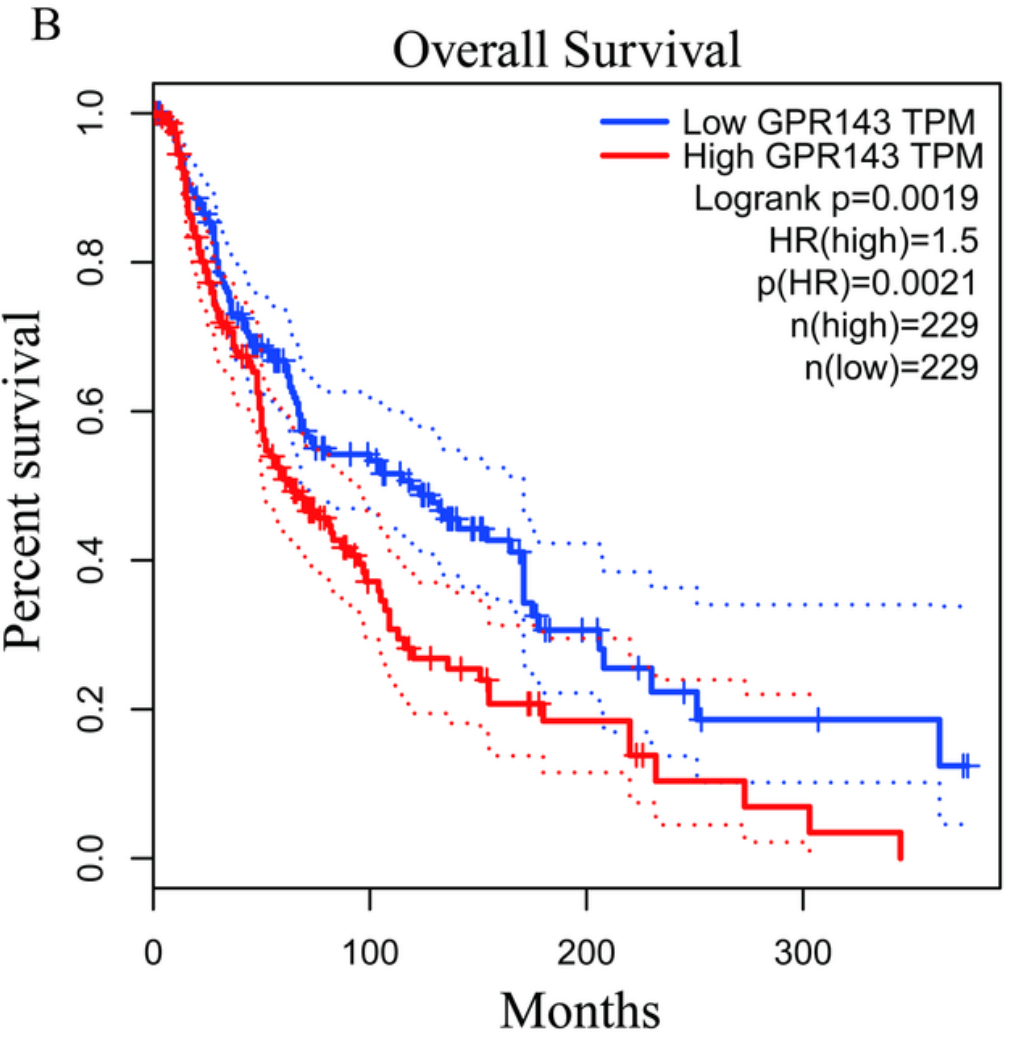

Figure 1

(a) Differential expression of GPR143 in different disease state (Tumor or Normal). (b) Survival curve of differential GPR143 expression were analyzed by GEPIA. 


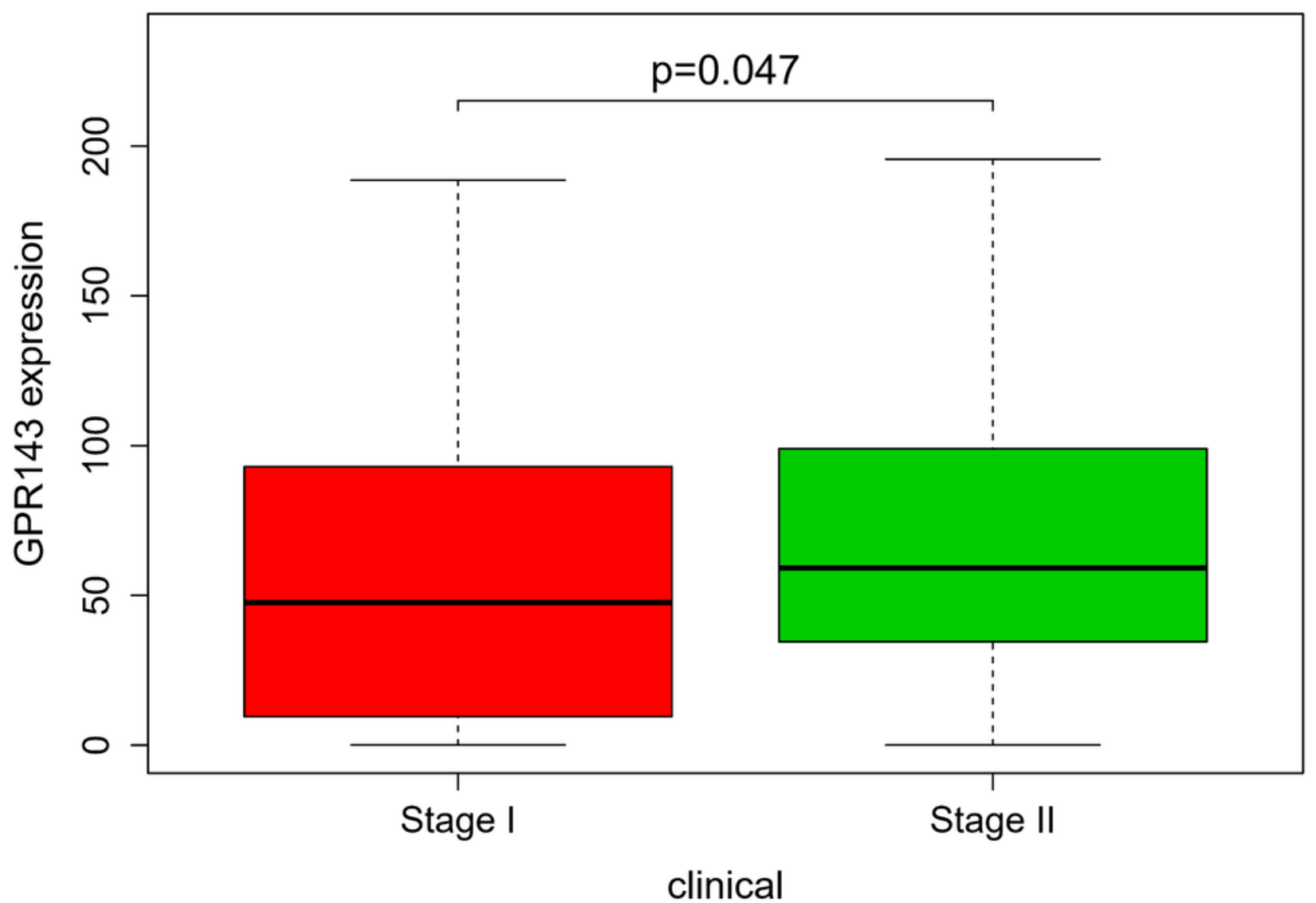

Figure 2

Differential expression of GPR143 in different cancer grade. (Based on the information of $77 \mathrm{~S} \rrbracket$ patients and $140 \mathrm{~S} \rrbracket$ patients in TCGA database). 


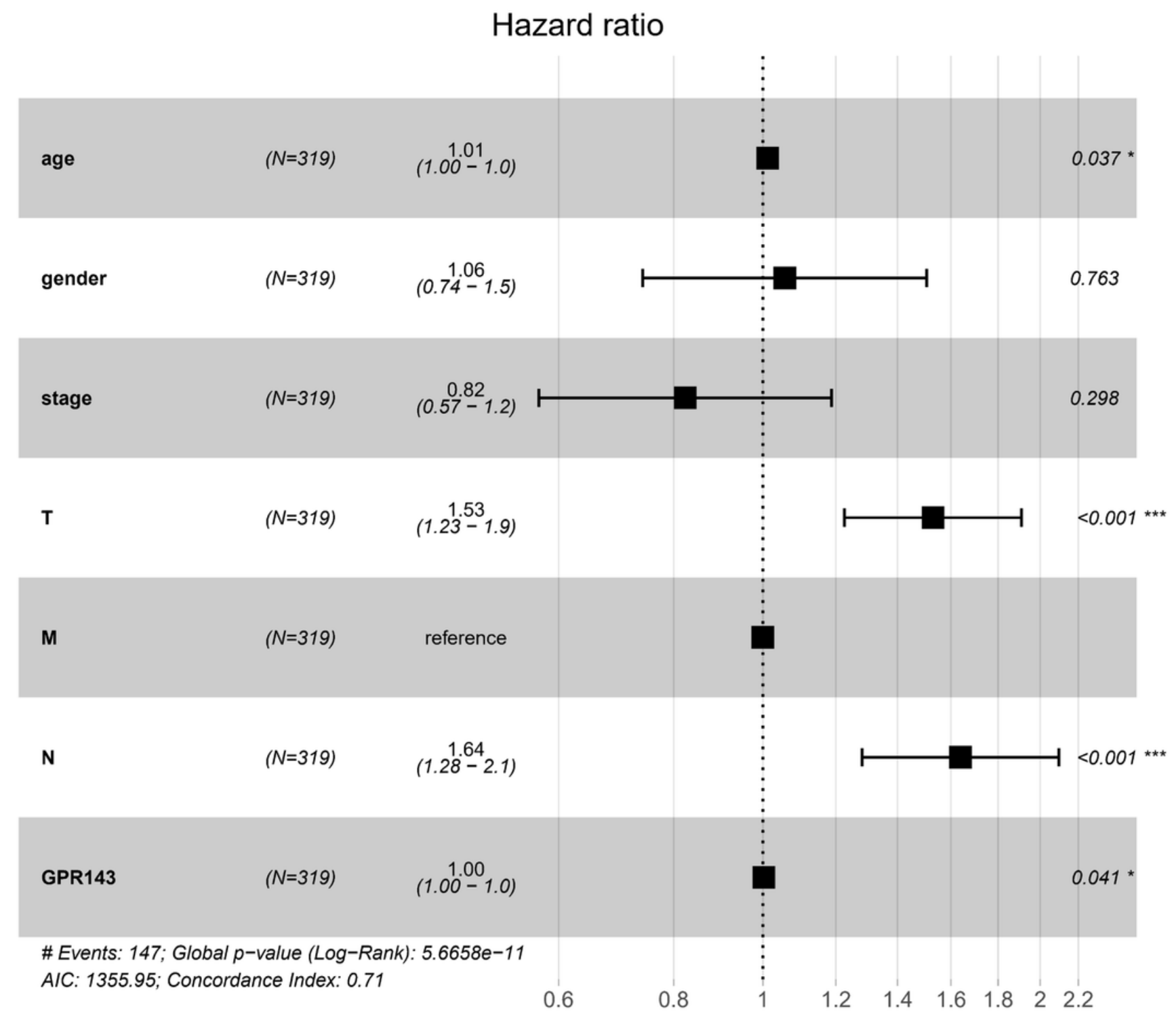

\section{Figure 3}

Multivariate Cox analysis of GPR143 expression and other clinical pathological factors. (As age, T (Stage of primary tumor), N (Stage of regional lymph node) and GPR143 expression are independent prognostic factors.) 
A

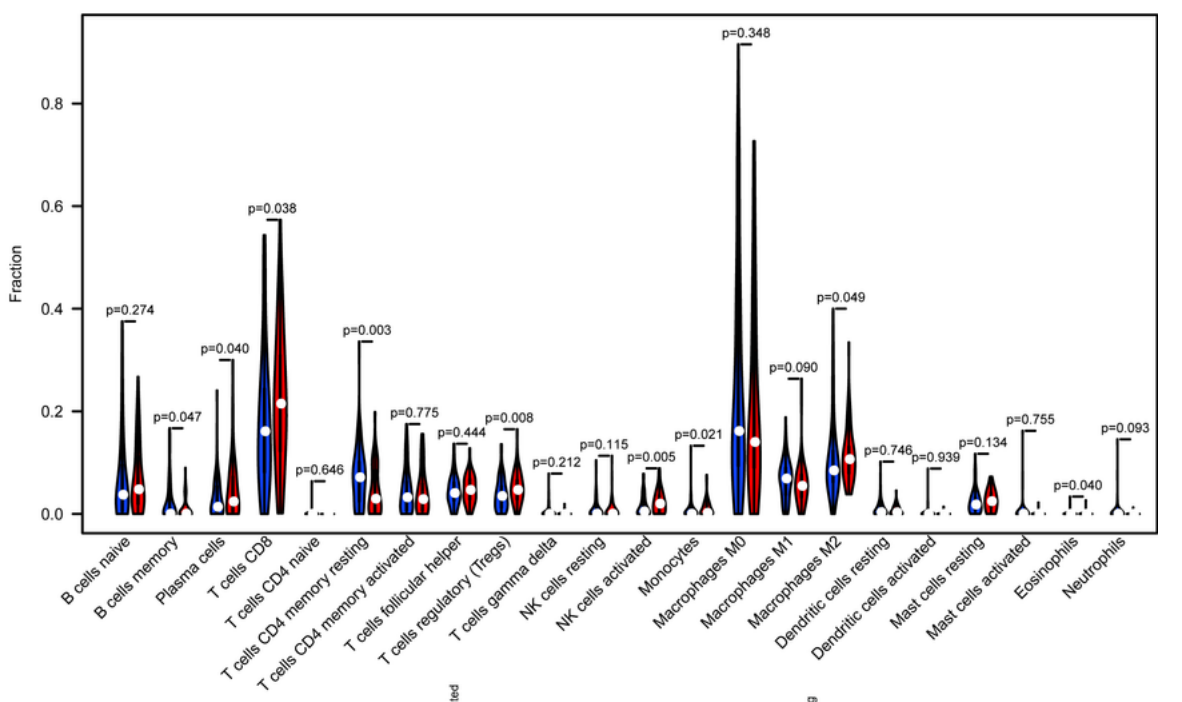

B

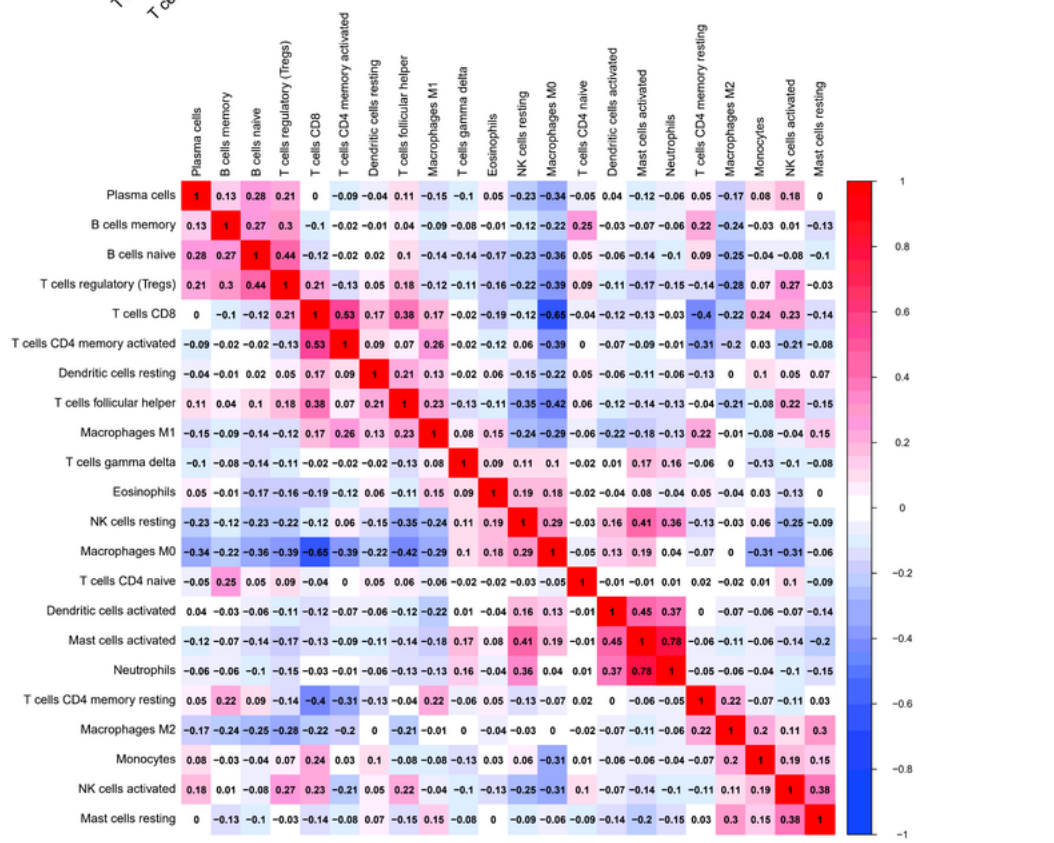

Figure 4

(a) The varied propotions of 22 subtypes of immune cells in high and low GPR143 expression groups in tumor samples. (b) Heatmap of 22 immune infiltration cells in tumor samples.

A
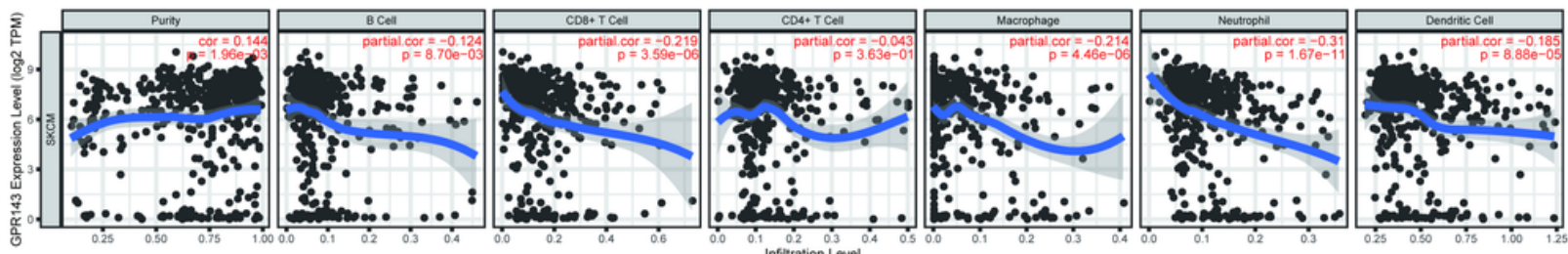

B

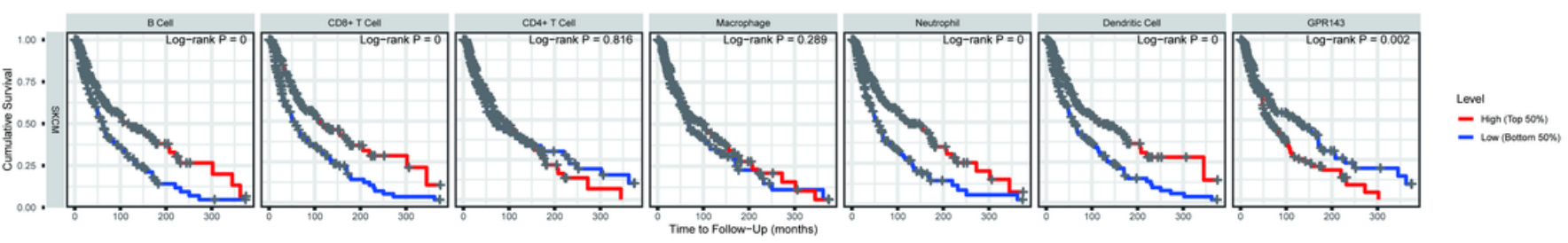




\section{Figure 5}

(a) GPR143 expression level has significant negative correlations with infiltrating levels of B cell, CD8+ T cells, CD4+ T cells, Macrophages, Neutrophils and DCs in SKCM. (b) cumulative survival is related to B cell, CD8+ T cells, Neutrophils and DCs in SKCM.

A
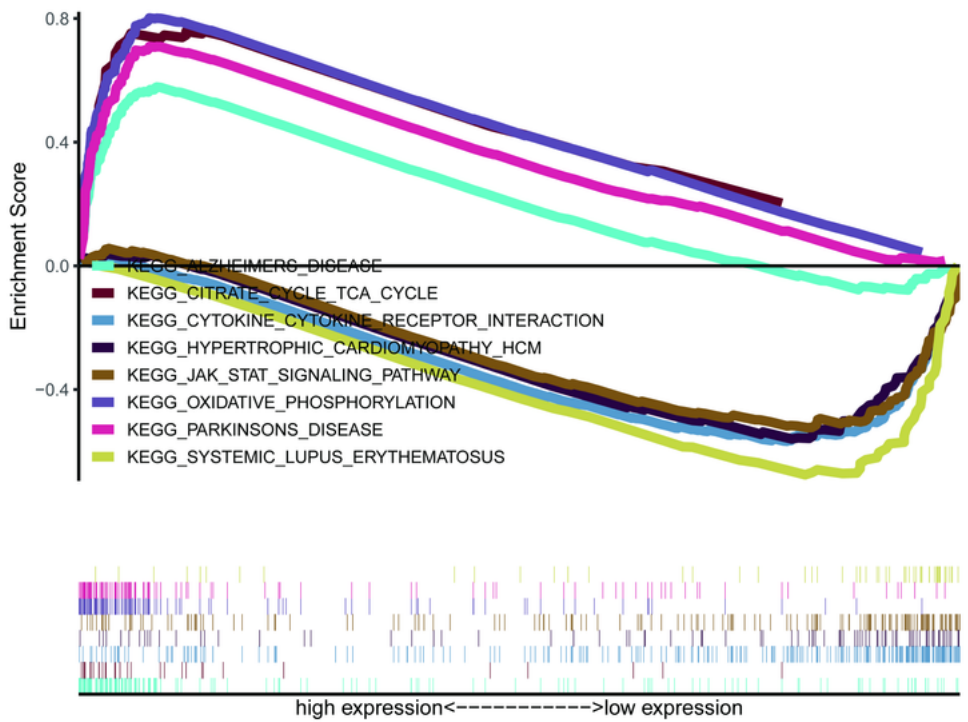

B
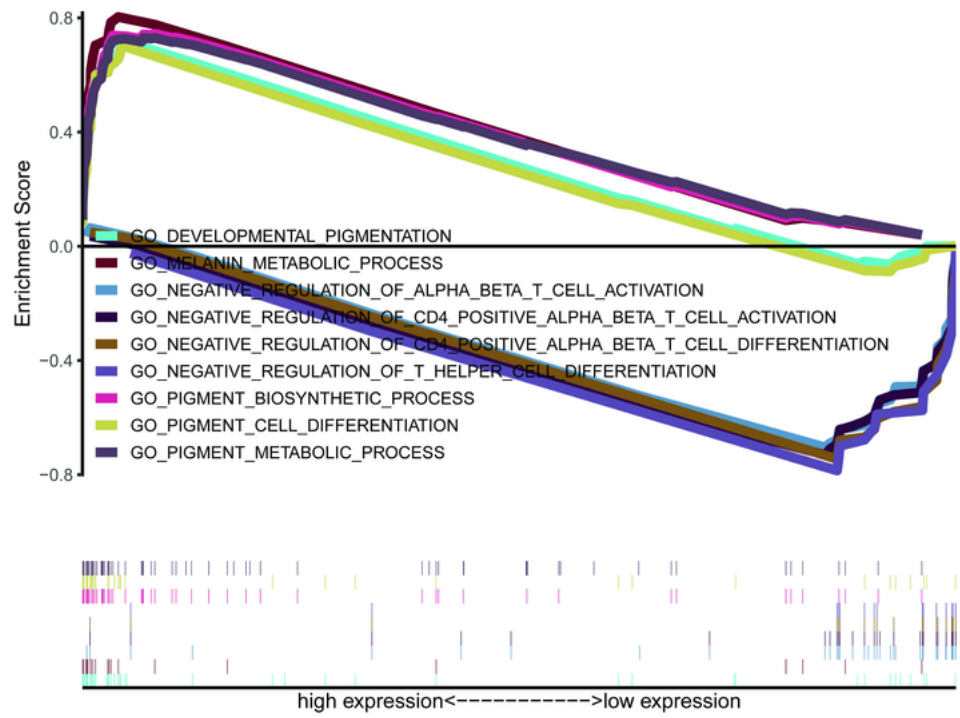

\section{Figure 6}

Enrichment plots from gene set enrichment analysis (GSEA). (a) GSEA results showing differential enrichment of genes in SKCM with high and low GPR143 expression. (b) GSEA results showing differential enrichment of genes in G0 with high and low GPR143 expression. 


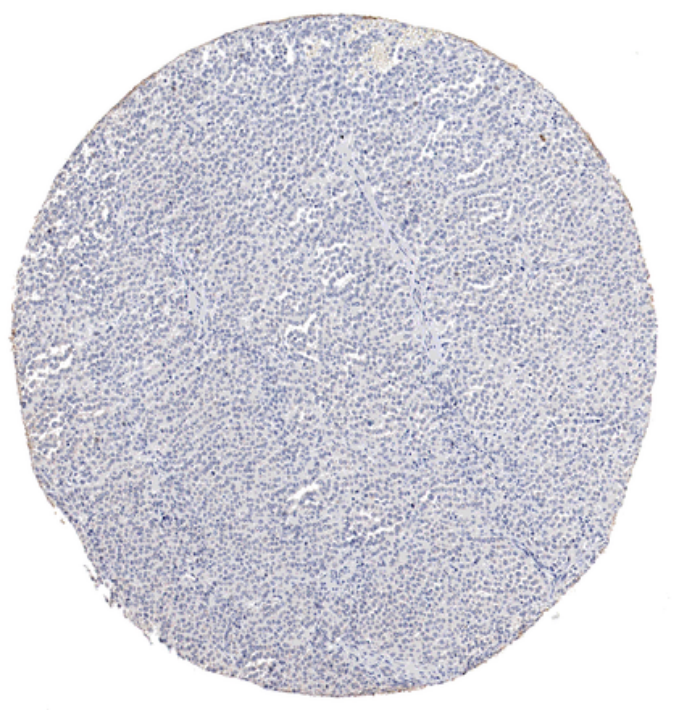

Normal

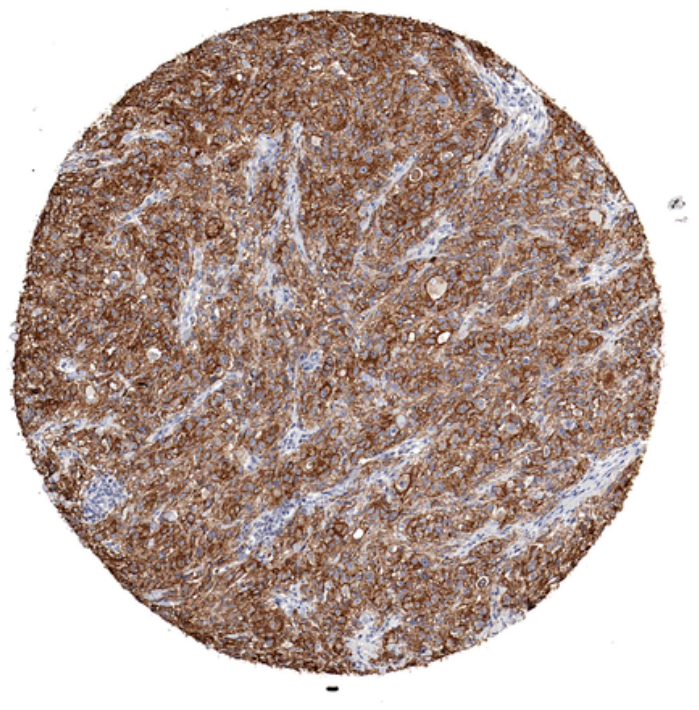

SKCM

\section{Figure 7}

Hepatic expression of GPR143 protein was visualized using immuohistochemistry via HPA. 